\title{
Full particle simulation of a perpendicular collisionless shock: A shock-rest-frame model
}

\author{
Takayuki Umeda ${ }^{1}$ and Ryo Yamazaki \\ ${ }^{1}$ Solar-Terrestrial Environment Laboratory, Nagoya University, Nagoya, Aichi 464-8601, Japan \\ ${ }^{2}$ Department of Physics, Hiroshima University, Higashi-Hiroshima, Hiroshima 739-8526, Japan
}

(Received May 25, 2006; Revised July 20, 2006; Accepted July 25, 2006; Online published October 13, 2006)

\begin{abstract}
The full kinetic dynamics of a perpendicular collisionless shock is studied by means of a one-dimensional electromagnetic full particle simulation. The present simulation domain is taken in the shock rest frame in contrast to the previous full particle simulations of shocks. Preliminary results show that the downstream state falls into a unique cyclic reformation state for a given set of upstream parameters through the self-consistent kinetic processes.
\end{abstract}

Key words: Collisionless shock, particle-in-cell simulation, shock rest frame.

\section{Introduction}

Collisionless shocks are universal processes in space and are observed in laboratory, astrophysical, and space plasmas, including astrophysical jets, an interstellar medium, the heliosphere, and the planetary magnetosphere. The classic picture of collisionless shocks is that they arise in a collisionless plasma streaming with a supersonic velocity against another collisionless plasma. The generation of plasma waves and turbulences, plasma heating and acceleration, and electromagnetic radiation processes at collisionless shocks are of great interest in astrophysics, solar physics, planetary physics, geophysics, and plasma physics. Detailed scientific issues in collisionless shock physics are described in Lembege et al. (2004). Kinetic simulations of collisionless shocks are essential approaches to solve these scientific issues.

There are several different methods for exciting collisionless shocks in numerical simulations of plasmas. The magnetic piston method (Lembege and Dawson, 1987a; Lembege and Savoini, 1992) is widely used in full particle simulations, in which a plasma is accelerated by an external current pulse applied at one side of the simulation domain. The simulation domain is taken in the upstream rest frame. The plasma is pushed by the "magnetic piston" into the background plasma, and the external pulse develops into a shock wave. Another method widely used is the injection method (Quest, 1985; Shimada and Hoshino, 2000; Schmitz et al., 2002a, b; Nishimura et al., 2003; Scholer et al., 2003; Lee et al., 2004), in which a plasma is injected from one side of the simulation domain and is reflected back when it reaches the other side. (Therefore this method is also called the reflection method or wall method.) The simulation domain is taken in the downstream rest frame, and a shock wave is

Copy right(c) The Society of Geomagnetism and Earth, Planetary and Space Sciences (SGEPSS); The Seismological Society of Japan; The Volcanological Society of Japan; The Geodetic Society of Japan; The Japanese Society for Planetary Sciences; TERRAPUB excited due to the interaction between the reflected and the injected plasma. Additional methods, such as the flow-flow method (Omidi and Winske, 1992) and the plasma release method (Ohsawa, 1985), are used in hybrid and full particle simulations. A detailed description of each method is given in Lembege (2003).

In these methods, an excited shock wave propagates upstream. Therefore, it is necessary to take a very long simulation domain in the propagation direction of the shock wave in order to study a long-time evolution of the excited shock wave. This makes it difficult to perform multidimensional simulations even with present-day supercomputers. In this letter, we first attempt to perform a full particle simulation of a collisionless shock in the shock rest frame: the collisionless shock is excited by using the "relaxation method" which was used in hybrid code simulations in 1980s (Leroy et al., 1981, 1982; Kan and Swift, 1983). This method has not been used in full particle simulations due to several difficulties in numerical techniques.

\section{Simulation Model}

Our simulation code (Umeda, 2004) is an improved version of one-dimensional Kyoto university electromagnetic particle code (Omura and Matsumoto, 1993), where Maxwell's equations and the relativistic equations of motion for individual electrons and ions are solved in a selfconsistent manner. The continuity equation for charge is also solved to compute the exact current density given by the motion of the charged particles (Umeda et al., 2003).

The simulation domain is taken in a one-dimensional system along the $x$-axis. The initial state consists of two uniform regions separated by a discontinuity. In the upstream region that is taken in the left hand side of the simulation domain, electrons and ions are distributed uniformly in $x$ and are given random velocities $\left(v_{x}, v_{y}, v_{z}\right)$ to approximate shifted Maxwellian momentum distributions with the drift velocity $u_{x 1}$, number density $n_{1} \equiv \epsilon_{0} m_{e} \omega_{p e 1}^{2} / e^{2}$, isotropic 
temperatures $T_{e 1} \equiv m_{e} v_{t e 1}^{2}$ and $T_{i 1} \equiv m_{i} v_{t i 1}^{2}$, where $m$, $e, \omega_{p 1}$, and $v_{t 1}$ are the mass, charge, upstream plasma frequency, and upstream thermal velocity, respectively. In this letter, subscripts " 1 " and " 2 " denote "upstream" and "downstream", respectively. The upstream magnetic field $B_{0 y 1} \equiv$ $-m_{e} \omega_{c e 1} / e$ is also assumed to be uniform, where $\omega_{c 1}$ is the upstream cyclotron frequency (with sign included). The downstream region taken in the right-hand side of the simulation domain is prepared similarly with the drift velocity $u_{x 2}$, density $n_{2}$, isotropic temperatures $T_{e 2}$ and $T_{i 2}$, and magnetic field $B_{0 y 2}$. In this letter we assume a perpendicular shock (i.e., $B_{0 x}=0$ ). As a motional electric field, an external electric field $E_{0 z}=-u_{x 1} B_{0 y 1}=-u_{x 2} B_{0 y 2}$ is also applied in both the upstream and downstream regions. At the left boundary of the simulation domain, we inject plasmas with the same quantities as those in the upstream region, while plasmas with the same quantities as those in the downstream region are also injected from the right boundary. We adopted absorbing boundaries to suppress the nonphysical reflection of electromagnetic waves at both ends of the simulation domain (Umeda et al., 2001).

In the present simulation, the time, velocity, and position are normalized by the initial upstream electron plasma frequency $\omega_{p e 1}=1.0$, upstream electron thermal velocity $v_{t e 1}=1.0$, and upstream electron Debye length $\lambda_{e 1} \equiv$ $v_{t e 1} / \omega_{p e 1}=1.0$, respectively. The initial temperatures in both upstream and downstream regions are assumed to be isotropic. In the upstream region, we assume a low beta and weakly magnetized plasma, such that $\beta_{e 1}=\beta_{i 1}=0.125$, and $\omega_{c e 1} / \omega_{p e 1}=-0.05$, which are similar to the recent full particle simulations (Shimada and Hoshino, 2000; Schmitz et al., 2002a, b; Lee et al., 2004). The light speed in the present simulation is given as $c=80.0$. The bulk flow velocity of the upstream plasma is assumed to be $u_{x 1}=4.0$, which corresponds to the Alfvén Mach number $M_{A}=10.0$. The ion-to-electron temperature ratio, $r_{T} \equiv T_{i} / T_{e}$ is assumed to be unity in the upstream region $\left(r_{T 1}=1\right)$. In addition to the upstream quantities $u_{x 1}, \omega_{p e 1}, \omega_{c e 1}, v_{t e 1}$, and $r_{T 1}$, we need the downstream ion-to-electron temperature ratio, $r_{T 2}$, so as to uniquely determine the other downstream quantities $u_{x 2}, \omega_{p e 2}, \omega_{c e 2}$, and $v_{t e 2}$ from the shock jump conditions for a magnetized two-fluid plasma consisting of electrons and ions with the equal bulk velocity and the equal number density. We adopt $r_{T 2}=4.0$ so that the thermal velocities of both downstream electrons and ions become much slower than the light speed. However, note that we can choose an arbitrary value for $r_{T 2}$. As can be seen later, the final cyclic reformation state does not depend on the choice of $r_{T 2}$. Since we performed the present simulation on a personal computer, we used a reduced ion-to-electron mass ratio $r_{m}=100$ for computational efficiency. With these parameters, we obtain the initial downstream quantities as $\omega_{p e 2}=1.95, \omega_{c e 2}=-0.19, u_{x 2}=1.05$, and $v_{t e 2}=7.55$.

We used 4096 cells for the upstream region and 8192 cells for the downstream region. The grid spacing and time step of the present simulation are $\Delta x / \lambda_{e 1}=1.0$ and $\omega_{p e 1} \Delta t=0.01$. We used 128 pairs of electrons and ions per cell in the upstream region and 512 pairs of electrons and ions per cell in the downstream region. It should be noted

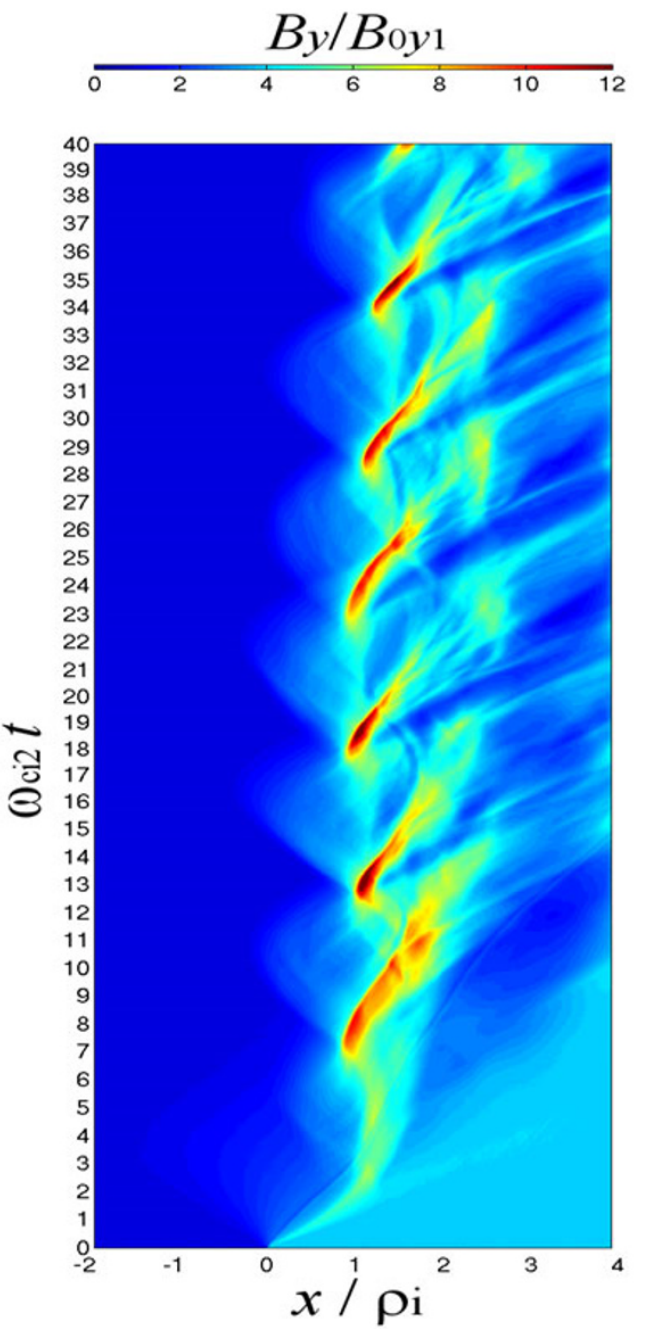

Fig. 1. The transverse magnetic field $B_{y}$ as a function of position and time. The time and position are normalized by $\omega_{c i 2}$ and $\rho_{i} \equiv u_{x 1} / \omega_{c i 2}$, respectively. The magnitude of magnetic field is normalized by $B_{0 y 1}$.

that such a few number of particles per cell is not enough to suppress the enhanced thermal fluctuations of particlein-cell codes. In the present simulation, however, the numerical noises due to random motions of individual particles are substantially reduced by adopting second-order schemes (Umeda, 2004).

\section{Simulation Result}

Figure 1 shows the transverse magnetic field $B_{y}$ as a function of position and time. The discontinuity exists at $x=0$ initially. However, the shock front shifts downstream due to the cyclotron motion of upstream ions that penetrate into the downstream region. A new shock front appears at $x / \rho_{i} \simeq 1$. The period from $\omega_{c i 2} t=0$ to 7 is the transition epoch from the initial to the cyclic reformation state (see below). It should also be noted that the initial discontinuity causes the non-physical generation of electromagnetic waves at the onset because Ampere's law $\frac{\partial B_{y}}{\partial x}=\mu_{0} J_{z}$ is not satisfied at the initial state. However, the electromagnetic waves are perfectly absorbed at both boundaries, and they never affect the simulation result in later time.

The typical processes for the self-reformation of perpen- 

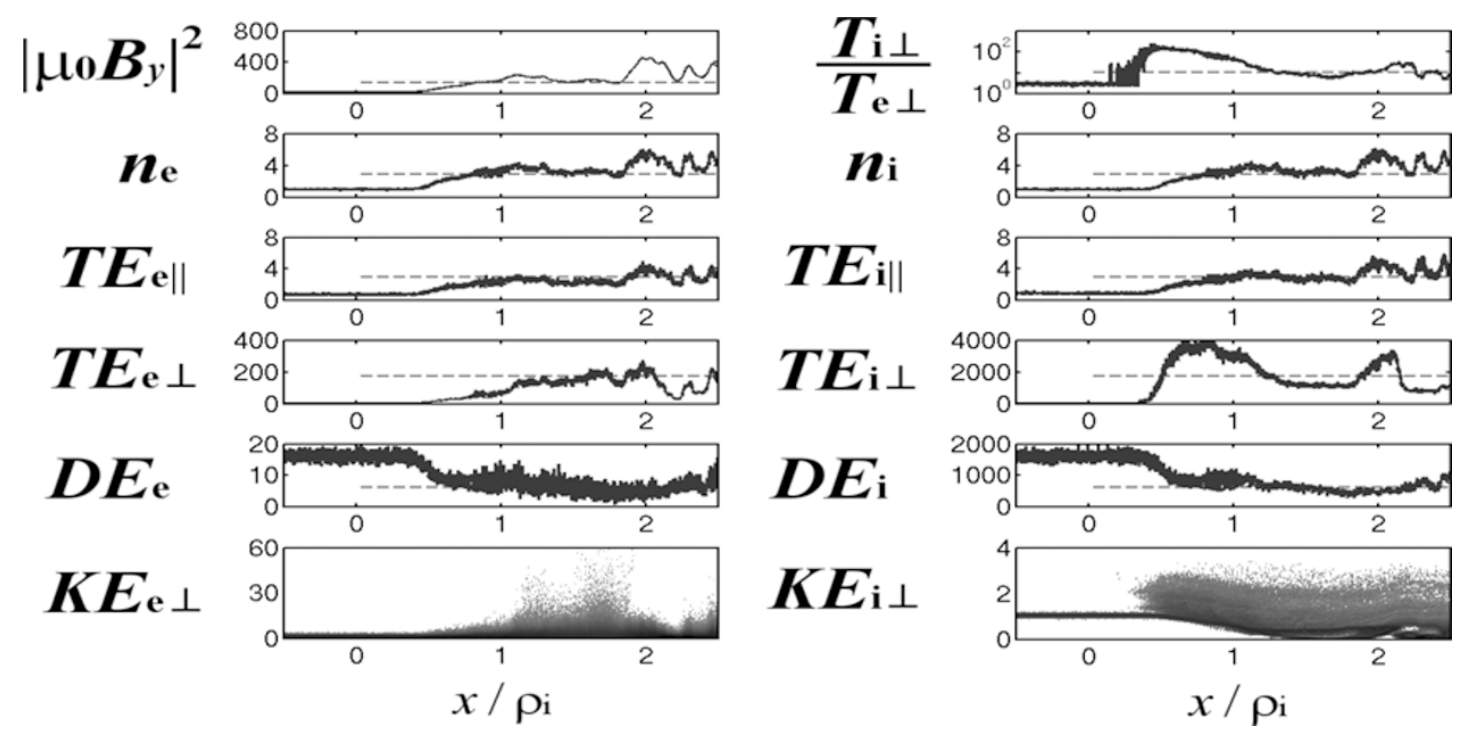

Fig. 2. Spatial profiles of the magnetic field energy density $\left|\mu_{0} B_{y}\right|^{2}$, ion-to-electron temperature ratio $r_{T}$, number densities $n$, thermal energy density components parallel and perpendicular to the ambient magnetic field $T E_{\|}$and $T E_{\perp}$, the drift energy density $D E$, and perpendicular kinetic energy versus position phase-space diagrams for electrons and ions at $\omega_{c i 2} t=38.1$. The number densities are normalized by the initial upstream electron density $n_{e 1}$ and the energy densities are normalized by the initial upstream thermal energy density of electrons $n_{e 1} m_{e} v_{t e 1}^{2}$. The dashed lines show the downstream values obtained from the shock jump conditions for anisotropic plasmas with the downstream quantities $v_{t e \| 2}=v_{t e 1}, r_{T \| 2}=r_{T 1}$, $r_{T \perp 2}=10$ and the shock speed $U / u_{x 1}=0.0282$. The perpendicular kinetic energies for electrons and ions are normalized by their upstream bulk energies, i.e., $\frac{1}{2} m_{e} u_{x 1}^{2}$ and $\frac{1}{2} m_{i} u_{x 1}^{2}$ respectively.

dicular collisionless shocks are as follows (Quest, 1985; Lembege and Savoini, 1992; Schmitz et al., 2002a, b; Nishimura et al., 2003; Hada et al., 2003). A portion of ions are reflected back from the shock ramp. The shock foot region broadens toward the upstream region as the reflected ions penetrate. However, the reflected ions return to the downstream region because of the cyclotron motion. The reflected ions can interact with upstream ions resulting in the self-formation of a new shock ramp in the old shock foot region. Finally, a new shock front appears at $x / \rho_{i} \simeq 1$, and ions are again reflected toward the upstream region from the new shock ramp.

The present simulation result shows that the timescale of the self reformation is almost equal to the cyclotron period of the downstream ions $\left(\omega_{c i 2} t \simeq 2 \pi\right)$, which is in agreement with the previous studies (Quest, 1985, Lembege and Savoini, 1992; Schmitz et al., 2002a, b; Nishimura et al., 2003; Scholer et al., 2003; Lee et al., 2004). Scholer et al. (2003) showed that the self reformation process is not a computational artifact with the real ion-to-electron mass ratio, while the ratio of electron plasma-to-cyclotron frequency is smaller $\left(\omega_{p e 1} / \omega_{c e 1}<10\right)$. On the other hand, Lee et al. (2004) generated the self reformation of more than six cycles, while their mass ratio is much smaller $\left(r_{m}=20\right)$. In the present shock-rest-frame model, we have also confirmed the shock reformation process up to six cycles with $r_{m}=100$, but with less grid cells.

In order to analyze the downstream condition in the cyclic reformation state, we plot in Fig. 2 spatial profiles of the magnetic energy density $\left|\mu_{0} B_{y}\right|^{2}$, the ion-to-electron temperature ratio perpendicular to the magnetic field $r_{T \perp}$, the number density $n$, parallel and perpendicular thermal energy density components $T E_{\|}$and $T E_{\perp}$, and the drift energy density $D E$ for electron and ions at $\omega_{c i 2} t=38.1$.

In the present shock-rest-frame model, a shock transition layer is self-consistently formed due to relaxation of the two plasmas with different quantities. The downstream region at the resulting cyclic reformation state is quite different from that of the initial state. For both electrons and ions, the spatial profiles of the thermal energy density component parallel to the ambient magnetic field are similar to those of number densities. This means that the downstream parallel temperatures for both electrons and ions become almost the same as those in the upstream region, i.e., $T_{e|| 1} \simeq T_{e|| 2} \simeq$ $T_{i \| 1} \simeq T_{i \| 2}$. On the other hand, the ratio of the ion-toelectron perpendicular temperature ratio is very large $(\sim$ $10^{2}$ ) in the transition region, and is typically $r_{T \perp 2} \sim 10$ in the downstream region $\left(r_{T \perp 2}=5.0 \sim 20.0\right)$.

As seen in Fig. 1, the excited shock wave propagates slowly downstream with the roughly estimated shock speed $U / u_{x 1}=0.0282$. The downstream electron and ion number densities in the cyclic reformation state become smaller than those at the initial state, whereas the downstream electron and ion bulk velocities become faster than those at the initial state. We performed several additional runs with different system sizes and found that the simulation resultsi.e., the shock speeds and the spatial profile of all physical quantities at an arbitrary time-are almost unchanged. In other words, the present system size is long enough to discuss the kinetic processes in the shock transition region.

In the downstream region of the cyclic reformation state, the physical quantities fluctuate and are not spatially uniform. We solved the shock jump conditions for anisotropic plasmas (Hudson, 1970) as a reference. Taking into account the shock speed and the typical downstream quantities $v_{t e|| 2}=v_{t e 1}, r_{T \| 2}=r_{T 1}$, and $r_{T \perp 2}=10$, the 
other downstream quantities are obtained as $\omega_{p e 2}=1.71$, $\omega_{c e 2}=-0.146, u_{x 2}=1.33$, and $v_{t e \perp 2}=5.42$. These quantities are plotted in Fig. 2 with dashed lines. A difference between the quasi-steady state by the shock jump conditions and the simulated downstream state is because of the dynamical shock reformation process. This might be another reason why we did not obtain the rigorous shock rest frame.

For a given set of upstream parameters, the fluid shock jump conditions cannot give the downstream state uniquely. In the electron-ion fluid, the total plasma pressure is defined as the sum of the electron and ion pressures, $P=n\left(T_{e}+T_{i}\right)$, and the shock jump conditions allow us to take an arbitrary downstream temperature ratio, $r_{T 2}$. However, the value of $r_{T 2}$ is determined by the kinetic dynamics as seen in the simulation result. We performed several additional runs with different initial downstream values and confirmed that the shock speed in our reference frame and downstream thermal properties at the cyclic reformation state do not depend on the initial value of $r_{T 2}$ but on the other initial downstream parameters, such as the magnetic field, the number density, the bulk velocity, and the total plasma pressure.

In the bottom panels of Fig. 2 we show perpendicular kinetic energy versus position diagrams for electrons and ions. We found that there exists a supra-thermal component of electrons at two local areas. The previous works reported the electron surfing acceleration with electrostatic solitary waves in the transition region (Hoshino and Shimada, 2002; Schmitz et al., 2002a, b). In the present simulation, we confirmed the existence of solitary waves in the shock foot region, where the maximum kinetic energy is about 30 -fold more than the initial kinetic energy. On the other hand, another supra-thermal component of electrons due to the ion deceleration is seen at the overshoot, where the maximum kinetic energy is much more ( $\left.>60 K E_{e 1}\right)$ than that of the non-thermal electrons via the surfing mechanism. Although this process was also found in the previous simulations (Hoshino and Shimada, 2002; Schmitz et al., 2002a, b), the maximum kinetic energy in the present simulation is more than that in their simulations. We expect that this process becomes more significant with a larger mass ratio.

\section{Conclusion}

We have developed a shock-rest-frame model for full particle simulations of perpendicular collisionless shocks based on the relaxation method used in the previous hybrid simulations (e.g., Leroy et al., 1981, 1982). We reconfirmed both the formation of microscopic solitary structures due to the current-driven instability and cyclic reformation for a long time with a much smaller simulation domain. The shock-rest-frame model allows us to perform multidimensional full particle simulations of planar shocks more readily with current supercomputers. We are extending the present shock-rest-frame model to oblique shocks. Preliminary results show that the present rest-frame model is very useful for exciting shock waves with arbitrary parameters.

Acknowledgments. The authors are grateful to F. Takahara, N. Okabe, and T. N. Kato for discussions. The computer simulation was performed as a collaborative research project at STEL in Nagoya University and at YITP in Kyoto University. This work was supported by Grant-in-aid for Encouragement of Young Scientists (B) \#18740153 from the Japan Ministry of Education, Culture, Sports, Science, and Technology (R. Y.).

\section{References}

Hada, T. et al., Shock front nonstationarity of supercritical perpendicular shocks, J. Geophys. Res., 108, 1233, doi:10.1029/2002JA009339, 2003. Hoshino, M. and N. Shimada, Nonthermal electrons at high Mach number shocks: Electron shock surfing acceleration, Astrophys. J., 572, 880 $887,2002$.

Hudson, P. D., Discontinuities in an anisotropic plasma and their identification in the solar wind, Planet. Space Sci., 18, 1611-1622, 1970.

Kan, J. R. and D. W. Swift, Structure of the quasi-parallel bow shock: Results of numerical simulations, J. Geophys. Res., 88, 6919-6925, 1983.

Lee, R. E. et al., Numerical simulations of local shock reformation and ion acceleration in supernova remnants, Astrophys. J., 604, 187-195, 2004.

Lembege, B. et al., Selected problems in collisionless-shock physics, Space Sci. Rev., 110, 161-226, 2004.

Lembege, B., Full particle electromagnetic simulation of collisionless shocks, in Space Plasma Simulations, edited by J. Buchner, C.T. Dum, and M. Scholer, pp. 54-78, Springer-Verlag Berlin Heidelberg, 2003.

Lembege, B. and J. M. Dawson, Plasma heating through a supercritical oblique shock, Phys. Fluids, 30, 1110-1114, 1987a.

Lembege, B. and J. M. Dawson, Self-consistent study of a perpendicular collisionless and nonresistive shock, Phys. Fluids, 30, 1767-1788, $1987 b$.

Lembege, B. and P. Savoini, Nonstationarity of a two-dimensional quasiperpendicular supercritical collisionless shock by self-reformation, Phys. Fluids B, 4, 3533-3548, 1992.

Leroy, M. M. et al., Simulation of a perpendicular bow shock, Geophys. Res. Lett., 8, 1269-1272, 1981.

Leroy, M. M. et al., The structure of perpendicular bow shocks, J. Geophys. Res., 87, 5081-5094, 1982.

Nishimura, K. et al., Particle simulations of re-formation at collisionless perpendicular shocks: Coherent behavior of reflected ions, J. Geophys. Res., 108, 1182, doi:10.1029/2002JA009671, 2003.

Ohsawa. Y., Strong ion acceleration by a collisionless magnetosonic shock wave propagating perpendicularly to a magnetic field, Phys. Fluids, 28, 2130-2136, 1985.

Omidi, N. and D. Winske, Kinetic structure of slow shocks: Effects of the electromagnetic ion/ion cyclotron instability, J. Geophys. Res., 97, 14801-14821, 1992.

Omura, Y. and H. Matsumoto, KEMPO1: Technical guide to onedimensional electromagnetic particle code, in Computer Space Plasma Physics, edited by H. Matsumoto and Y. Omura, 487 pp, Terra Scientific, Tokyo, 1993.

Quest, K. B., Simulations of high-Mach-number collisionless perpendicular shocks in astrophysical plasmas, Phys. Rev. Lett., 54, 1872-1874, 1985 .

Schmitz, H. et al., The influence of electron temperature and magnetic field strength on cosmic-ray injection in high Mach number shocks, Astrophys. J., 570, 637-646, 2002a.

Schmitz, H. et al., Electron preacceleration mechanisms in the foot region of high Alfvenic Mach number shocks, Astrophys. J., 579, 327-336, 2002b.

Scholer, M. et al., Quasi-perpendicular shocks: Length scale of the crossshock potential, shock reformation, and implication for shock surfing, J. Geophys. Res., 108, 1014, doi:10.1029/2002JA009515, 2003.

Shimada N. and M. Hoshino, Strong electron acceleration at high Mach number shock waves: Simulation study of electron dynamics, Astrophys. $J .$, 543, L67-L71, 2000.

Umeda, T., Study on nonlinear processes of electron beam instabilities via computer simulations, Ph.D. Thesis, Kyoto University, 2004.

Umeda, T. et al., An improved masking method for absorbing boundaries in electromagnetic particle simulations, Comput. Phys. Commun., 137, 286-299, 2001.

Umeda, T. et al., A new charge conservation method in electromagnetic particle simulations, Comput. Phys. Commun., 156, 73-85, 2003.

T. Umeda (email: umeda@stelab.nagoya-u.ac.jp) and R. Yamazaki (email: ryo@theo.phys.sci.hiroshima-u.ac.jp) 